\title{
The Effect of PTEN on Apoptosis in NSCLC Cell Line ${ }^{\dagger}$
}

\author{
Aydin Demiray ${ }^{1}$, Hakan Akca ${ }^{1}$ and Buket Er Urganci $2, *$ \\ 1 Department of Medical Genetics, Faculty of Medicine, Pamukkale University, 20070 Denizli, Turkey; \\ ademiray@pau.edu.tr (A.D.); hakca@pau.edu.tr (H.A.) \\ 2 Department of Medical Biology, Faculty of Medicine, Pamukkale University, 20070 Denizli, Turkey \\ * Correspondence: aber@pau.edu.tr \\ † Presented at the 2nd International Cell Death Research Congress, Izmir, Turkey, 1-4 November 2018. \\ Published: 6 December 2018
}

\begin{abstract}
PTEN is inactivated in a subset of lung cancer; therefore, we investigated the involvement of PTEN inactivation in invasiveness of lung cancer cells. AKT at Ser473 was phosphorylated in several lung cancer cell lines with loss of PTEN expression. Therefore, we created a tetracycline inducible expression system of wild-type PTEN (PTEN-WT) as well as catalytically (PTEN-G129R) and lipid phosphatase (PTEN-G129E) inactive PTEN mutants using the PC14, lung adenocarcinoma cell lines, in which endogenous PTEN expression was not detected and AKT at Ser473 was phosphorylated by Western blot analysis. These results suggest that the PTEN gene cannot induce apoptosis alone, but apoptosis can be achieved by the suppression of other pathways. The absence of PTEN expression gives an invasive and metastatic phenotype to cancer cells, and also while staging of cancer cells that allows the transition to a further stage of cancer.
\end{abstract}

Keywords: PTEN; apoptosis; lung cancer

\section{Introduction}

Lung cancer is the leading cause of cancer-related deaths. $80 \%$ of lung cancers are non-small cell lung (NSCLC) cancers [1]. While NSCLC shows approximately $65 \%$ heterogeneity, $45 \%$ is adeno and squamous type NSCLC [2]. While surgical interventions are performed for patients with stages 1 and 2 , some of stage 3 patients have undergone surgical intervention and some of them have received radiotherapy and chemotherapy [3]. Nevertheless, 3-year life expectancy is $35-50 \%$ for stage 2 patients and $28 \%$ for stage 3 patients, while 5 -year survival is $15 \%$ in NSCLC patients [4]. In other words, survival rate, chemotherapy and radiotherapy application at early stage patients, increase the survival rate. Tumor cells have an aggressive character while escaping from apoptosis. Two protein families are involved in the apoptotic process; Bcl-2 family and caspases. The Bcl-2 family consists of two opposing groups, one prevents the apoptotic process while the other induces. There is a balance between these two groups in a normal cell. Epidermal Growth Factor Receptor (EGFR) is a transmembrane protein. When EGFR receptor stimulated with cytokines, forms a dimer by autophosphorylation. When EGFR receptor stimulated, it activates the PI3K/AKT/Nf-kB pathway with grb-sos adapter protein and then the BAD is phosphorylated and the cell escapes from apoptosis. The PTEN tumor suppressor protein dephosphorylation blocks the PI3K/AKT phosphorylation step. PTEN is a tumor suppressor gene located at the 10q23 region of the 10th chromosome and double acting phosphatase as lipid phosphatase and protein phosphatase. Lipid phosphatase activity is higher than protein phosphatase activity. PTEN is a tumor suppressor gene that controls the cell cycle, plays a role in the control of proliferation and apoptosis. PTEN promoter region is hypermethylated in many cancers. According to the literature, the two-point mutation in the GAA sequence of the 129th amino acids of the PTEN cDNA significantly affects the PTEN activity. The mutation that converts PTEN G 129R to arginine (AGA) instead of 129. amino acid glycine 
(GAA), creates catalytically inactive PTEN. The mutation that converts PTEN G129E to glutamic acid (GAA) instead of 129th amino acid glycine (GAA), causes the loss of lipid dephosphorylase effect of PTEN only [2]. Thus, these mutant PTEN forms allow researchers to understand the pathway mechanisms of PTEN. In our study, we examined the effect of PTEN expression on apoptosis by transfecting the Teton system in NSCLC cell line.

\section{Materials and Methods}

PC14 cells were cultured in RPMI-1640 medium (Sigma Aldrich, St. Louis, MO, USA) supplemented with $10 \%$ fetal bovine serum (Invitrogen, Carlsbad, CA, USA) at $37^{\circ} \mathrm{C}$ in a humidified incubator with $5 \% \mathrm{CO}_{2}$.

The expression vectors used in the study are "Teton" system vectors (Invitrogen, Carlsbad, CA, USA). These vector systems allow expression of the cloned gene only in the cell by the addition of tetracycline. Furthermore, these vector systems are suitable for tumor suppressor gene expression and function studies that can be performed at any time. PTEN cDNA was amplified using forward (5'-CGCGAATTCGCCATGGCAGCCATCATCAAAGAGATCGTTAGCAGAAACAAAAGGAGA TATCAAGAGGATGGATTCGACTTAGAC-3') and reverse (5'-CGCGAATTCTCAGACTTTTGTA ATTTGTGTATGCTGATCTTCATCAAAAGGTTCATTCTCTGGATCAGAGTCAGTGGAGGTGTC AGA-3'). primers and Pfu DNA polymerase (Thermo Fisher Scientific, Rockford, IL, USA), and amplified cDNA was cloned into pcDNA 3 and a tetracycline-inducible vector pcDNA4/TO. The PTENG129R and PTENG129E mutants were generated by site-directed mutagenesis. The PTENwt, PTENG129R and PTENG129E expression vectors cloned. By using FUGEN (Roche), PC14 cells were transfected with the muck vector (pcDNATETON), PTENwt (pcDNATETONPTENwt), PTEN G129E (pcDNATETON-PTENG129E) and PTEN G129R (pcDNATETON-PTEN G129R) expression vectors. PC-14 cells were co-transfected with tet 6 and tet 4 vector systems. After $24 \mathrm{~h}$ of transfection, the medium was treated with blastocidin $(100 \mu \mathrm{g} / \mathrm{mL})$ and zeocin $(10 \mu \mathrm{g} / \mathrm{mL})$. The selection was continued for 26 days, while the medium was freshened every three days. Twenty-five days after, the PTENwt, PTENG129R, and PTENG129E vectors transfected colonies were identified.

Cell lysates were prepared in ice-cold RIPA buffer (10 mM Tris-HCl pH7.5, $150 \mathrm{mM} \mathrm{NaCl}, 2 \mathrm{mM}$ EDTA, $1 \%$ NP40, $1 \%$ sodium deoxycholate, $0.1 \%$ SDS). Cellular debris was removed by centrifugation at $12,000 \times \mathrm{g}$ for $5 \mathrm{~min}$ at $4{ }^{\circ} \mathrm{C}$. One hundred $\mathrm{g}$ of proteins were subjected to SDS-PAGE using 2-15\% or $7.5 \%$ polyacrylamide gels (Pierce, Thermo Fisher Scientific, Rockford, IL, USA), proteins were immunoblotted onto Hybond-PVDF membrane (Amersham-PharmaciaBiotech, Piscataway, NJ, USA) and labeled with antibodies. PTEN and GAPDH, primary antibodies were obtained from Santa Cruz Biotechnology (Dallas, TX, USA). Primary and secondary antibody labeling blots were treated with Super Signal West Pico chemiluminescent substrate (Pierce, Thermo Fisher Scientific, Rockford, IL, USA), exposed to Hyperfilm ECL (Amersham-PharmaciaBiotech, NJ, USA) and developed.

Mock, pcDNA4/TO-PTEN-WT, pcDNA4/TO-PTEN-G129R, and pcDNA4/TO-PTEN-G129E transfected PC14 cells were seeded into 96-well plates $\left(1 \times 10^{5} \mathrm{cells} / \mathrm{mL}\right)$ and treated with tetracycline $(2 \mu \mathrm{g} / \mathrm{mL})$ for $0,24,48$ and $72 \mathrm{~h}$. At the end of the incubation period, apoptotic cell numbers were counted by Terminal Deoxynucleotidyl Transferase (TdT)-mediated dUTP nick end labeling (TUNEL) assay (Invitrogen, Carlsbad, CA, USA). Paired $t$-test and multivariable test were used for statistical analysis. SPSS 17th package program was used for this process.

\section{Results}

Western blot analysis was performed in order to understand whether PTEN wt, PTENG129R, and PTENG129E transfected PC 14 cells reaffirmed PTEN expression. Muck vector, PTENwt, PTENG129R, and PTENG129E transfected PC 14 cells were incubated for $2 \mathrm{~h}$ with/without $2 \mu \mathrm{g} / \mathrm{mL}$ tetracycline and divided into two groups. After incubation, cells were lysed with RIPA buffer. PTEN labeling indicates that the transfectant PC 14 cells express PTEN wt and mutant forms (Figure 1). The same result also indicates that PTEN expression presence with tetracycline incubation. 


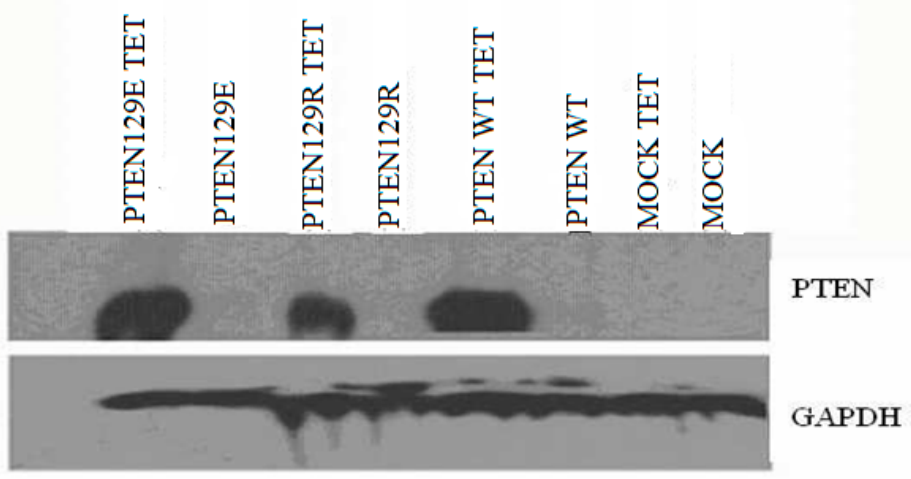

Figure 1. Expressions of PTEN on PC14 cells transfected with Mock, PTENwt, PTENG129R and PTENG129E vectors.

The effect of PTEN expressions on apoptosis, in muck vector, PTENwt, PTENG129R, and PTENG129E transfected PC 14 cells was also investigated by Terminal deoxynucleotidyl transferase (TdT)-mediated dUTP nick end labeling (TUNEL) assay (Figure 2). The difference between apoptotic cell number of muck vector and mutant PTEN vector transfected PC14 cells was not statistically significant $(p: 0.89, p>0.05)$.

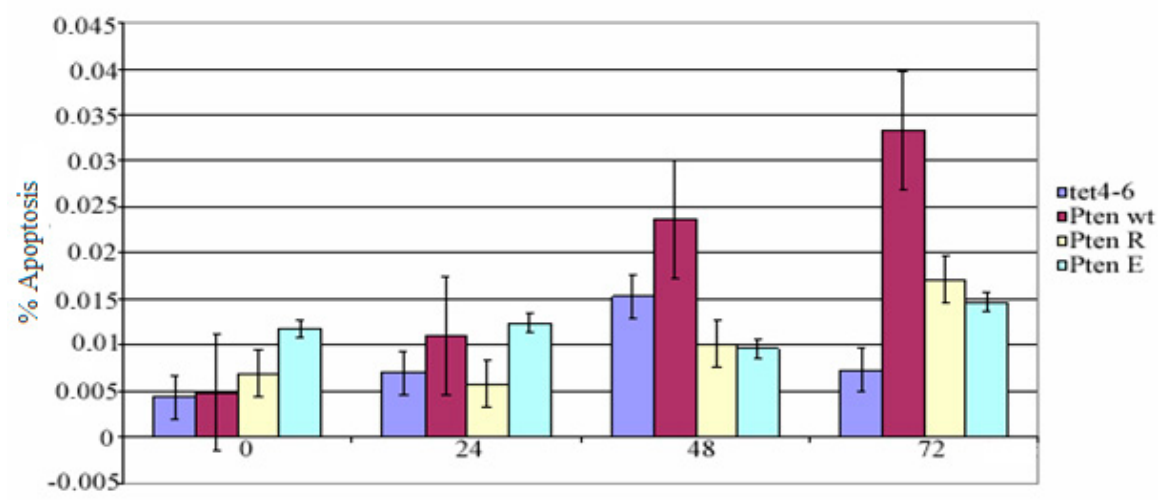

Figure 2. The apoptotic cell count of TET4-6, PTENwt, PTEN G129E and PTEN G129R transfected PC 14 cells between $0-72 \mathrm{~h}$.

\section{Discussion}

In many non-small cell lung cancers, the PTEN gene cannot be expressed as a result of deletion or promoter methylation. The non-small cell lung cancer cell line (NSCLC) we used in our study, PC 14 cells, can not express the PTEN gene because of the methylated promoter. In our study, PTEN wt, PTENG129R, and PTENG129E have successfully transfected into NSCLC cell line PC 14 cells and the expression was indicated by western blot analysis. Phosphated (active) Akt causes escape from apoptosis. Also, there is study reporting that PTEN gene expression supports apoptosis [5]. However, in our study, the difference between apoptotic cell number of muck vector and mutant PTEN vector transfected cells was not statistically significant. However, some studies have shown that apoptosis in PTEN wt transfected cells is only stimulated by radiotherapy [6]. These results suggest that the PTEN gene cannot induce apoptosis alone, but apoptosis can be achieved by the suppression of other pathways. Furnari et al. (1998) reported that PTEN wt, PTENG129R, and PTENG129E transfection did not induce apoptosis in the glioma cells [7]. This study supports our results. The absence of the expression of PTEN leads to the grading of cancer in prostate cancers as an aggressive (severe) tumor from a moderate tumor condition [8]. The absence of PTEN expression gives an invasive and metastatic phenotype to cancer cells, and also while staging of cancer cells that allows the transition to a further stage of cancer. PTEN cannot induce apoptosis alone. 


\section{References}

1. Ohashi, R.; Takahashi, F.; Cui, R.; Yoshioka, M.; Gu, T.; Sasaki, S.; Tominaga, S.; Nishio, K.; Tanabe, K.K.; Takahashi, K. Interaction Between CD44 and Hyaluronate Induces Chemoresistance in Non-small Cell Lung Cancer. Cell Cancer Lett. 2007, 252, 225-234.

2. Kandasamy, K.; Srivastava, R.K. Role of the Phosphatidylinositol 3 Kinase/PTEN/Akt Kinase Pathway in Tumor Necrosis Factor-related Apoptosis- Inducing Ligand-Induced Apoptosis in Non-Small Cell Lung Cancer Cells. Cancer Res. 2002, 62, 4929-4937.

3. Lim, W.T.; Zhang, W.H.; Miller, C.R.; Watters, J.W.; Gao, F.; Viswanathan, A.; Govindan, R.; Mcleod, H.L. PTEN and Phosphorylated AKT Expression and Prognosis in Early- and Late-Stage Non-Small Cell Lung ancer. Oncol. Rep. 2006, 17, 853-857.

4. Tang, J.M.; Hea, Q.Y.; Guob, R.X.; Chang, X.J. Phosphorylated Akt Overexpression and Loss of PTEN Expression in Non-small Cell Lung Cancer Confers Poor Prognosis. Lung Cancer 2006, 51, 181-191.

5. Han, S.W.; Ritzenthaler, J.D.; Zheng, Y.; Roman, J. PPAR $\gamma / \beta$ Agonist Stimulates Human Lung Carcinoma Cell Growth Through Inhibition of PTEN Expression: The Involvement of PI3K and NF-kB Signals. J. Physiol. Lung Cell Mol. Physiol. 2008, 294, 1238-1249.

6. Pappas, G.; Zumstein, L.A.; Munshi, A.; Hobbs, M.; Meyn, R.E. PTEN Expression Radiosensitizes Nonsmall Cell Lung Cancer Cells by Suppressing DNA Repair Capacity. Cancer Gene Ther. 2007, 14, 543-549.

7. Furnari, F.B.; Huang, H.-J.S.; Cavenee, W.K. The Phosphoinositol Phosphatase Activity of PTEN Mediates a Serum-Sensitive G1 Growth Arrest in Glioma. Cells Cancer Res. 1998, 58, 5002-5008.

8. Shukla, S.; MacLennan, G.T.; Hartman, D.J.; Fu, P.; Resnick, M.I.; Gupta, S. Activation of PI3K-Akt Signaling Pathway Promotes Prostate Cancer Cell Invasion. Int. J. Cancer 2007, 121, 1424-1432.

(C) 2018 by the authors. Licensee MDPI, Basel, Switzerland. This article is an open access article distributed under the terms and conditions of the Creative Commons Attribution (CC BY) license (http://creativecommons.org/licenses/by/4.0/). 